\title{
A New Variable Temperature Solution-Solid Interface Scanning Tunneling Microscope
}

\author{
Abdolreza Jahanbekam $^{1}$, Ursula Mazur ${ }^{1}$ and K. W. Hipps ${ }^{1}$ \\ 1. Department of Chemistry and Materials Science and Engineering Program, Washington State \\ University, Pullman, Washington, 99164-4630, USA
}

Scanning tunneling microscopy (STM) has been widely used to investigate surface structures and electronic properties of adsorbed species on surfaces and also for observing chemical reactions on surfaces. The technique can be used in various environments such as vacuum, air and solution. Among these environments, ultrahigh vacuum (UHV)-solid and solution-solid (SS) interfaces get the most attention. If we compare sample preparation procedures for these two environments, it is much easier to prepare samples and take measurements at the SS interface, although STM images in UHV generally show higher resolution. If the sample cannot be evaporated without decomposition, SS interface studies may be the only choice. Generally, studies in UHV require that the molecules of interest be vapor deposited - which is impossible for many compounds. No matter how the sample is prepared, by the nature of the experiment, UHV studies are not compatible with chemical equilibrium involving material transport to and from the surface. SS interface studies, on the other hand, are easily adaptable to equilibrium studies. In SS studies, one can change the solvent in order to tune molecule-solvent and substrate-solvent interactions at the SS interface, thereby changing the ordering and structure of the adsorbed species. Gyarfas et al. demonstrated that the length of the alkane chain in different alkanoic solvents had a role in determining the surface structure of coronene on gold [1]. The repair of defects in self assembled layers at the SS interface can be promoted if there is a dynamic exchange between molecules on the surface and in the solution phase [2].

Different areas of technology and science profit from temperature dependent SS interface studies. In the area of technology, these studies can yield vital insights into the critical problems in catalysis, spincasting, friction, crystallization and organic electronics. Temperature dependent studies can yield a great wealth of information including diffusion rates, reaction rates, activation energies and thermodynamic quantities such as entropy and enthalpy of adsorption and/or surface reaction. Because different surface species reach equilibrium at different temperatures, and because some surface reactions are kinetically controlled, a study of a given solution-surface pair as a function of temperature can lead to the discovery of new materials and phases. While STM imaging studies of the SS interface have been in existence for more than two decades, temperature dependent studies have been rare. But one may ask why this fruitful branch of science is seldom seen in the scientific literature. The answer can be traced to the imposed limitation by the design of the commercial STM instruments. In the conventional "in air" design of systems for temperature dependent study, only the sample is heated. This is not the most desirable design for SS interface work. In fact, there are critical problems that are associated with this design. To solve these problems we have designed and built a new system in which all mechanical components of the STM system and the sample are heated in a controlled environment at the saturation vapor pressure of the solution under study (Figure1). This enables the user to image at the SS interface with some nonconducting volatile solvents for long periods of time ( $>24$ hours) which opens up another exciting door to this area of science.

In order to fabricate the instrument we married an improved version of a commercially available STM head with a novel environmental chamber and an external preamplifier system of our own design. We have successfully tested our system by performing a temperature dependence study of cobalt(II) 
octaethylporphyrin (CoOEP) at the toluene/Au(111) interface. It is demonstrated that the lattice parameters remain constant within experimental error from $24{ }^{\circ} \mathrm{C}$ to $75{ }^{\circ} \mathrm{C}$. We report the unit cell of CoOEP at the toluene/ $\mathrm{Au}(111)$ interface (based on two molecules per unit cell) to be $\mathrm{A}=(1.36 \pm 0.04)$ $\mathrm{nm}, \mathrm{B}=(2.51 \pm 0.04) \mathrm{nm}$ and $\alpha=97^{\circ} \pm 2^{\circ}$.

We are also using this STM to study the competitive adsorption of a two component solute system at the solution-solid interface. In general, upon introduction of a multi-component solution to a substrate several scenarios are possible. Phase segregation, formation of randomly mixed monolayers, and coadsorption of components in an ordered fashion are among possible situations. As a model system, competitive adsorption of CoOEP and coronene molecules at the phenyloctane/Au(111) interface is being studied. We find that at room temperature CoOEP cannot be displaced from the gold surface by exposure to a near saturation solution of coronene. On the other hand, coronene is readily completely replaced by CoOEP near saturation solutions. We will report on the concentration and temperature dependence of the competitive adsorption/desorption and how it may lead to two or more phases present on the gold surface.

References:

[1] B. J. Gyarfas et al, Langmuir 21, 919 (2005).

[2] M. Hibino et al, Thin solid films 273, 272 (1996).

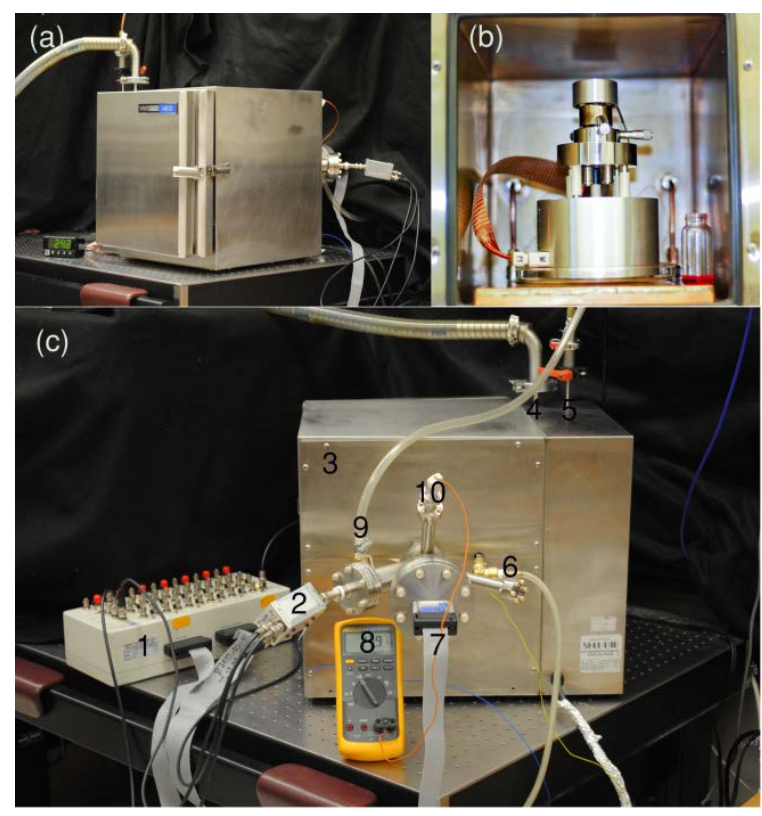

Figure 1. (a) Front view of controlled-temperature and controlled-atmosphere chamber. (b) Inside view of the chamber showing the STM assembly and a solution reservoir. (c) Backside view of the chamber with all of the connections. The parts are -- 1: Signal access module, 2: Pre-amplifier, 3: Chamber, 4: Port for evacuating the chamber, 5: Port for introducing gas into the chamber, 6: Outlet for fluid cooling, 7: Interface cable between STM and controller, 8: Digital voltmeter for reading thermocouple temperature, 9: Inlet for fluid cooling, 10: Thermocouple connection ports. 